\section{Restoration of Consciousness with Zolpidem in a Patient with Sporadic Creutzfeldt - Ja- kob Disease (sCJD)}

Roshan Sutar ${ }^{1 *}$, Abhinav Nahar ${ }^{1}$, Seby Kurthukulangara', Ravinder Jeet Singh Sidhu' ${ }^{2}$, Harish $T^{1}$, Jitender Saini ${ }^{3}$, Ravi Yadav $^{2}$ and Santosh Chaturvedi ${ }^{1}$

${ }^{1}$ Department of Psychiatry, National Institute of Mental Health and Neurosciences, Bangalore, India

${ }^{2}$ Department of Neurology, National Institute of Mental Health and Neurosciences, Bangalore, India

${ }^{3}$ Department of Neuroradiology, National Institute of Mental Health and Neurosciences, Bangalore, India

\begin{abstract}
Creutzfeldt-Jakob Disease (CJD) is a rare form of dementia with rapid progression and spongiform degeneration of brain secondary to accumulation of misfolded prion proteins. Sporadic CJD presents with rapidly progressing cognitive decline, myoclonus, motor and behavioural disturbances. As the disease advances, consciousness is progressively impaired; different pharmacological agents and deep brain stimulation have been tried to improve consciousness with minimal evidence. Zolpidem is a non-benzodiazepine hypnotic but also has an arousal action and has been reported as an "awakening drug" in some patients with disorders of consciousness. We report a patient with SCJD whose consciousness markedly improved with a zolpidem.
\end{abstract}

Keywords: Consciousness; Creutzfeldt - Jakob disease; Zolpidem

\section{Introduction}

Creutzfeldt - Jacob disease is a rare form of dementia associated with neuropathological finding of accumulation of prion proteins in the brain with rapid deterioration and disability [1]. Alteration in the level of consciousness has been studied in dementia and other hypoxic brain injuries. Attempt to modulate the effect of pharmacological agents and deep brain stimulation to attain the maximum level of consciousness is ongoing but the evidence is lacking [2]. This report supports the use of zolpidem in the arousal from altered consciousness in a patient with sporadic CJD.

*Corresponding author: Roshan Sutar, Department of Psychiatry, National Institute of Mental Health and Neurosciences, Bangalore, India, Tel: +91 9980590172; E-mail: roshidoc@yahoo.co.in

Citation: Sutar R, Nahar A, Kurthukulangara S, Sidhu RJS, Harish T, et al. (2016) Restoration of Consciousness with Zolpidem in a Patient with Sporadic Creutzfeldt - Jakob Disease (sCJD). J Addict Addictv Disord 3: 010.

Received: June 13, 2016; Accepted: August 24, 2016; Published: September 08, 2016

Copyright: @ 2016 Sutar R, et al., This is an open-access article distributed under the terms of the Creative Commons Attribution License, which permits unrestricted use, distribution, and reproduction in any medium, provided the original author and source are credited.

\section{Case Report}

A 59 year old woman presented with 7 months history of gradual onset visual disturbances which progressed to near complete visual loss (diagnosed as cortical blindness). She also developed behavioural changes characterized by reduced spontaneity, decreased social interaction, and progressive reduction in speech output, along with worsening memory for recent events and gait ataxia. Two weeks prior to admission, she became progressively mute with poor comprehension, and also developed double incontinence, generalised myoclonic jerks, was bed - bound and was unable to take foods orally. There was no history of seizures and no past history of tissue or organ transplantation or eating bovine brain. There was no family history of dementia or neurological illnesses except Parkinsonism in patient's elder brother.

On examination she was awake and was opening eyes spontaneously with no verbal output. She was not obeying simple commands but would localize pain. She had severe rigidity of all limbs (lower limbs slightly more than upper limbs), intermittent myoclonic jerks which were not stimulus sensitive, and brisk deep tendon reflexes with bilaterally extensor plantar reflex. Grasp reflexes were present bilaterally.

Investigations including $\mathrm{CBC}$, metabolic panel, thyroid profile and serum B12 levels were normal. TPO antibody, autoimmune encephalitis workup for antibodies against NMDA, AMPA 1 and 2, VGKC (LGI-1 and CASPR), GABA 1 and 2, and autoimmune panel for vasculitis were negative. MRI Brain (Figure 1) showed cortical ribbon sign with bilateral involvement of caudate and putamen, which was highly suggestive of prion disease like CJD. EEG showed frequent bilateral periodic triphasic discharges with diffuse slowing of background activity. Patient met diagnostic criteria for probable CJD by World Health Organization (Figure 2).

Patient had not responded earlier to trials of L-dopa, donepezil, memantine, amantadine and a course of methyl prednisolone. Care givers reported earlier noticing a marked improvement in her responsiveness when she was given tablet zolpidem for insomnia for two nights. Based on this history, a trial of immediate release tablet zolpidem $5 \mathrm{mg}$ HS was started. Response was noticed 7 hours after the first dose in the form of patient spontaneously getting up from the bed, attempting to walk with assistance and taking food orally by self-removal of nasogastric tube. She also started initiating verbal communication with care givers. This response was maintained on regular dosing of zolpidem $5 \mathrm{mg}$ BD without any noticeable side effects. There was no further requirement of nasogastric tube feeding. She was shortly discharged with advice to continue Tab zolpidem $5 \mathrm{mg}$ $\mathrm{BD}$. She did not come for follow up. A telephonic enquiry 3 months later revealed that, after a few days of sustained improvement, she again progressively deteriorated to a vegetative state despite taking zolpidem, which was then discontinued.

\section{Discussion}

In our patient, clinical, electrophysiological and radiological features supported diagnosis of SCJD, which is thought to have an irreversible, rapidly progressive course [3]. Deterioration in the levels of consciousness during disease progression was a prominent feature in our patient [4]. 

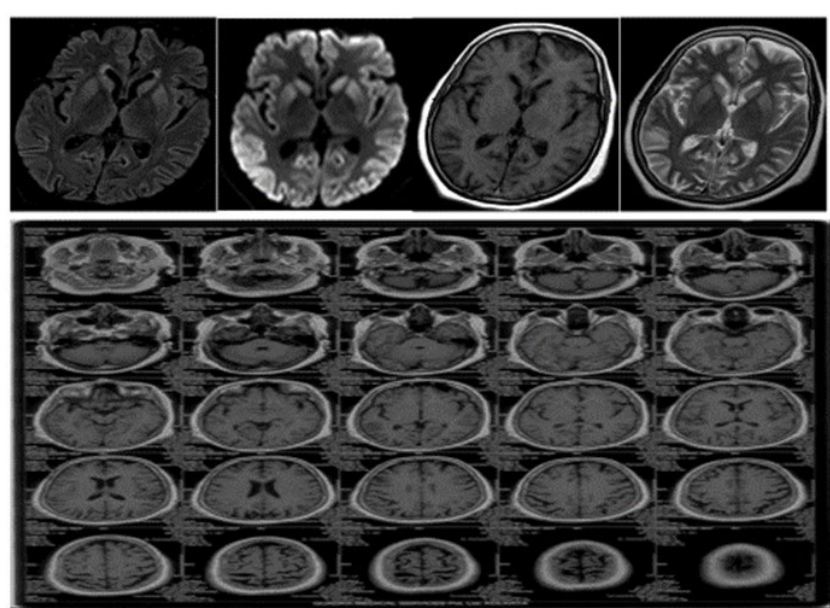

Figure 1: MRI brain picture showing cortical ribbon sign and bilateral involvement of caudate and putamen in our patient with sporadic Creutzfeldt - Jakob disease.

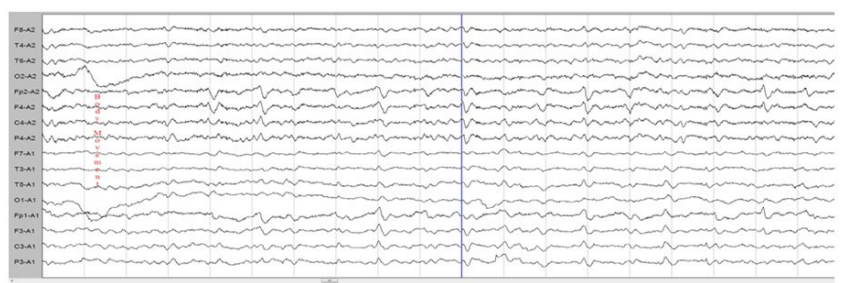

Figure 2: Phase lag response in temporal and occipital leads of EEG in CJD.

Zolpidem preferentially acts on omega 1-GABA-A receptors within the GABA receptor - chloride channel complex. Arousal appears to be specific to zolpidem unlike other non - specific benzodiazepines due to possible differential receptor affinity, but exact mechanism is still unclear. One hypothesis is that reduced arousal in patients following brain disease leads to alteration in the number of GABA receptors as a mechanism of neuroprotection. Zolpidem appears to reverse it by reactivation of dormant brain neural networks. Another hypothesis claims that zolpidem may activate limbic loops modulating motivational processes thus leading to disinhibition and thalamocortical overactivity [5]. Paradoxical restoration of consciousness by CNS depressants is thought to be by functional restoration of certain networks like reticular activating system, cortico - cortical loop and cortico - thalamo-cortical loop as suggested by functional neuroimaging studies $[3,6,7]$. However, not all patients with disorders of consciousness respond to zolpidem as noted in a placebo controlled trial [2].

In conclusion, zolpidem appears to have a role in improving consciousness in some patients with disorders of consciousness such as CJD. Though response is likely to be only for a short period, the very fact that improvement albeit transient is possible, in rapidly progressive neurodegenerative diseases otherwise deemed to be irreversible, merits further studies which may also enhance our understanding of neurobiology of consciousness [7].

\section{Acknowledgements}

We thank to Department of Neurology, Neuro-radiology and Consultation liaison psychiatry for assistance in writing this report.

\section{Conflict of Interest}

None

\section{References}

1. Kojima G, Tatsuno BK, Inaba M, Velligas S, Masaki K, et al. (2013) Creutzfeldt-Jakob disease: a case report and differential diagnoses. Hawaii $J$ Med Public Health 72: 136-139.

2. Whyte J, Myers R (2009) Incidence of clinically significant responses to zolpidem among patients with disorders of consciousness: a preliminary placebo controlled trial. Am J Phys Med Rehabil 88: 410-418.

3. Brefel-Courbon C, Payoux P, Ory F, Sommet A, Slaoui T, et al. (2007) Clinical and imaging evidence of zolpidem effect in hypoxic encephalopathy. Ann Neurol 62: 102-105.

4. Whyte J, Rajan R, Rosenbaum A, Katz D, Kalmar K, et al. (2014) Zolpidem and restoration of consciousness. American Journal of Physical Medicine \& Rehabilitation 93: 101-113.

5. Lo YL, Tan EK, Ratnagopal P, Chan LL, Tan TE (2008) Zolpidem and its effects on hypoxic encephalopathy. Ann Neurol 64: 477-478.

6. Singh R, McDonald C, Dawson K, Lewis S, Pringle AM, et al. (2008) Zolpidem in a minimally conscious state. Brain Inj 22: 103-106.

7. Clauss RP, Nel WH (2004) Effect of zolpidem on brain injury and diaschisis as detected by $99 \mathrm{mTc}$ HMPAO brain SPECT in humans. Arzneimittelforschung 54: 641-646. 